\title{
Solving the traveling salesman problem using genetic algorithms with the new evaluation function
}

\author{
Hamid Tabatabaee*, Hosian Salami, Nafiseh Sedaghat, Naghmeh Sharif
}

\begin{abstract}
Traveling salesman problem is of the known and classical problems at Research in Operations. Many scientific activities can be solved as traveling salesman problem. Existing methods for solving hard problems (such as the traveling salesman problem) consists of a large number of variables and constraints which reduces their practical efficiency in solving problems with the original size. In recent decades, the use of heuristic and meta-heuristic algorithms such as genetic algorithms is considered. Due to the simple structure of metaheuristic algorithms that have shown greater ability is more used by researchers in operational research. In this study, the improved genetic algorithm is used to solve TSP that the difference of it with the standard genetic algorithm is in the evaluation function. The new evaluation function is from a common evaluation function and a new idea.
\end{abstract}

Keywords - Travelling Salesman problem, genetic algorithms, problems of NP-Hard, NP-Complete, Hamiltonian cycle

\section{Introduction}

The travelling salesman problem in the 18th century by the Irish mathematician named Sir William Rowam Hamilton and the British mathematician named Thomas Penyngton Kirkman was studied. It is believed that the overall shape of the TSP was studied firstly by Kalr Menger in Vienna and Harvard[1]. The main motivation for the study on the TSP does not arise directly from the application, but also stems from this fact that this problem provides an ideal platform for studying the general methods which can be applied to a wide range of discrete optimization problems[2]. The idea of problem is to find the shortest path of sellers with starting from a specific city, meeting once an $\mathrm{n}$ city and eventually coming back to the city of origin. The question that arises here is that, in what order should we meet the cities so that the minimum distance is?

Hamid Tabatabaee

Department of Computer Engineering, Quchan Branch, Islamic Azad University, Quchan,

Iran.

Hosian Salami

Department of Computer Engineering, Ferdows higher education institute Mashhad, Iran

\section{Nafiseh Sedaghat}

Young Researchers and Elite Club, Mashhad Branch, Islamic Azad University, Mashhad,

Iran.

Naghmeh Sharif

Social Insurance Organization, Branch 3, Mashhad,

Iran
This problem plays as a sub-problem in many applications of transport. For example, the issue of setting school bus routes for students to ride in an area, visiting the gas turbine engine, computer wiring, the timing of the delivery of food to those with disabilities, the timing of cranes in warehouses, setting routes for trucks to remove the post packages. Although the applications of transportation are the most natural TSP applications, because the model is simple it would lead in a large number of attractive applications in other areas. A classic example, the timing of machine for drilling holes in the circuit boards, in which drilling holes ,cities and when to move the tip of drill from a hole to next one is the cost of travel. TSP has special significance because a large number of practical applications can be demonstrated in this way.

In addition, it has a significance of theory in complexity theory because TSP is one of the classes of combinatorial optimization problems of NP-Complete that is of the hard optimization problems, because the set of solutions of possibility that satisfy the conditions of problem but not necessarily optimal, are finite, although they are usually very large[2]. So it has this feature that progress on it leads to the development on combinatorial optimization problems. So far, a lot of efforts to find a reasonable way to solve this problem have been proposed which can be roughly divided into two categories: general and detailed procedures. The exact algorithms typically have very high computational time and it is impractical to use them to great things, therefore, the use of approximate methods is more applicable. In the category of approximate methods, approaches using random search terms such as genetic algorithms are easier and provide better results, so the algorithms used in numerous articles and it has been focused on various aspects of them. In this paper, we aim to introduce a new evaluation function in genetic algorithms to solve the traveling salesman problem. According to the authors' knowledge, this paper is the first attempt to focus more on the evaluation function to solve this problem. The rest of the contents of this article are as follows. Part 2 focuses on the definition of the traveling salesman problem. In the third part we have a review of earlier work. In Section 4 , the proposed method has been introduced. We will review the test results at the Section 5, at end of Section 6 Conclusions and future work will be dealt. 


\section{Defining the problem}

The travelling salesman problem is shown with full graph with weighted edges $\mathrm{G}=(\mathrm{V}, \mathrm{E})$ that $\mathrm{V}$ is a set of $\mathrm{n}$ node or vertex $(n=|V|)$ that indicates cities and $\mathrm{E} \subseteq \mathrm{V} \times \mathrm{V}$ is a set of edges or directed edges. To each edge $(i, j) \in E$ the length of $\mathrm{djj}$ that is the distance between the cities of $\mathrm{I}$ and $\mathrm{j}$ that is $\mathrm{i}$, $\mathrm{j} \in \mathrm{V}$, is attributed. The travelling salesman problem can be naturally Symmetric or asymmetric. In the asymmetric travelling salesman problem (aTSP), the distance between the nodes of $\mathrm{I}$ and $\mathrm{j}$ is dependent on edge scan direction, at least one edge $(\mathrm{i}, \mathrm{j})$ that there is $\mathrm{d}_{\mathrm{ij}} \neq \mathrm{d}_{\mathrm{ji}}$. In the symmetric travelling salesman problem, for all of the edges there is the equation $d_{i j}=d_{j j}$ in $E$. the aim of the travelling salesman problem is to find Hamiltonian cycle with the least length of graph. Hamiltonian cycle is a closed path that all of the nodes of $\mathrm{G}$ are met only once. So, an optimal solution for travelling salesman problem is permutation of $\pi$ from the nodes with index of $\{1, \ldots \ldots, n\}$ so that the length of $f(\pi)$ is least, that $\mathrm{f}(\pi)$ is measured by the following formula:

$$
f(\pi)=\sum_{i=1}^{n-1} d_{\pi(i) \pi(i+1)}+d_{\pi(n) \pi}
$$

\section{Earlier works}

One of the first applications of dynamic programming, is Held-Karp algorithm that solves TSP problem in time $\mathrm{O}(\mathrm{n})$. Other approaches include various types of algorithms of branching and restrictions, the gradual recovery algorithms that apply the techniques are reminiscent of linear programming. To achieve the optimal solution to the problem is of inefficient aspects of computing. By taking $n$ as the number of cities to be visited, will be the number of possible routes that cover all cities (n-1)! /2 (and thus the number of possible solutions to TSP). For problems with large size, it is almost impossible to obtain the Maintaining the Integrity of the Specifications optimal solution in a reasonable time. The context for the development and application of approximation algorithms, or heuristic has been created. Never approximation methods that do not guarantee the optimal solution, however, close to optimal solutions are provided in a reasonable time. Heuristic algorithms are available in different categories that we can mention the Heuristics to create or improve net. Different heuristics for the net include nearest neighbor heuristic, greedy, etc. Heuristics to create net after finding a net, do not try to improve it, instead, after production a net with a heuristic to create, tries to improve their nets have been found. Heuristic exchange 2-opt and 3-opt and the k-opt this purpose have been proposed[1]. Heuristics of exchange of 2opt and 3-opt and the k-opt have been proposed for this purpose.

Along with heuristic algorithms, a large number of searches have used random searching such as banned research[4], genetic algorithms, ant colony algorithm[5,10], PSO algorithm[11,12]. A comparison between a numbers of random search algorithms can be found in [13].
For some reasons as inherently parallel algorithm, global search and a simple implementation, genetic algorithm is now one of the most random search algorithms[14]. Genetic Algorithm first proposed by Holland in 1975[15]. The genetic algorithm is based on Darwin rules about 'survival of the fittest'. Under this rule the fittest people is more likely to survive and more likely that their good genes to be transferred to the next generation. In GA, each individual (or chromosome that is part of the population) indicates a possible solution to this problem.

The first scholar that used genetic algorithm to overcome the problem of TSP was Brady[16]. Many articles have used genetic algorithms to solve this problem. The documents focus on genetic algorithm not only to improve the quality of the final results, but also try to reduce the time of calculation of genetic algorithm [14].

Often these documents focus on some aspects of genetic algorithms such as how to display solutions (chromosomes), operators[17,23] and selection methods. In order to completely examine the different types of displaying solutions as well as cutting and mutation operators that have been used to solve this problem in the genetic algorithm[24] you can refer to. In [27] three methods of selection of tournament, roulette wheel and roulette wheel based ranking were compared to solve this issue and on the basis of test results stated that the selection tournament creates better results stated that the selection of tournament has better results than other two methods. [25] And [26] an overview of some of the efforts to solve this problem with genetic algorithms has been done.

Due to the relatively slowness of GA, certain documents dealt with improving the computation time of the algorithm. In algorithm [14] PREGA is presented, which aims to eliminate duplicate computations and is based on reducing the example. The idea Beyond the PREGA is identifying common genes in all chromosomes in early generations to eliminate duplicate computations.

The combination of genetic algorithm with other methods is also widely used. In the [27] combination of genetic algorithms and local search methods for solving optimization problems has been studied. The authors in the various forms, have examined the complementary roles that can have use of local search methods to improve performance of genetic algorithm, and the process of genetic algorithm that can use them,. The [28] authors focus on the combination of genetic algorithm and dynamic programming to solve the problem TSP and various articles that have used this combination have been examined. GA combination with other methods of random search is also of other approaches that have been used in various articles. Cuckoo search [29] method with genetic algorithm in order to avoid exposure to local minimums has been used. In [30] with GACO algorithms that combines genetic algorithm and ant colony is used to solve the TSP problem.

As mentioned, existing efforts to improve the genetic algorithm to solve TSP problem have studied various aspects of it but there is no attempt to evaluate the effect on the results. To achieve this goal, in this paper we have introduced the results of a new evaluation function to solve the problem. The results showed performance improvements 
using the new evaluation function compared to traditional evaluation function.

\section{Iv. The proposed method}

In genetic algorithms there are lots of cases such as problem encoding, how to select the initial population, population size, the maximum number of generations, the standard stop, the possibility of cutting the risk of mutation, the function evaluation, elitism, operator selection, cutting operator, operator mutations that selection of these affects the performance of GA. In the following sections, the configuration used in genetic algorithms has been proposed.

\section{A. Problem encoding}

At this stage, we make decisions about how chromosomes are shown. Choosing a bad way can lead to exposure of GA in a local minimum. Using binary representation that consists of bits 0 and 1 because of the extreme magnitude and complexity of the checking credit of produced chromosomes at the end of each generation is impractical. However, if all the above problems are overcome, the convergence rate is very low and the solutions obtained is not optimal. The method used here is the symbolic display for each chromosome [31]. In this method, we show each chromosome with a one-dimensional array of integers between 1 and $\mathrm{n}$. In other words each

\section{B. Selection of initial population}

The initial population is randomly selected. Accordingly, for producing each chromosome in the population, permutation of numbers from 1 to the number of cities is produced.

Since the assumption is that all cities are connected to each other, producing all chromosomes are valid.

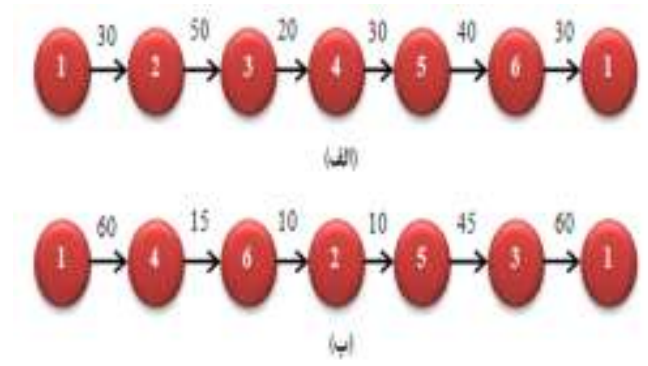

Figure 1-Comparison of two chromosomes with net length of 200

\section{C. evaluation function}

At GA, we need to a measure to compare our chromosomes accordingly and recognize their optimality. The criteria for each chromosome in TSP are equal to the cost of avoiding that the chromosome shows.

According to the model previously mentioned, if the cost matrix is shown with $D$, then $D_{i j}$ represents the cost of the chromosome represents a cycle and any gene represents a city.

With these definitions, the following mathematical model for TSP can be made:

Suppose that $C=\{1,2, \ldots i, \ldots n\}$ are the cities that are met. Therefore, each chromosome can be demonstrated as follows:

$$
\mathrm{X}=\left(\mathrm{X}_{1}, \mathrm{X}_{2}, \ldots \mathrm{X}_{\mathrm{i}}, \ldots \mathrm{X}_{\mathrm{n}}\right)
$$

A round of chromosomes can be represented as follows:

$$
\mathrm{X}_{1} \rightarrow \cdots \rightarrow \mathrm{X}_{\mathrm{i}} \cdots \rightarrow \mathrm{X}_{\mathrm{n}} \rightarrow \mathrm{X}_{1}
$$

In this model, each chromosome to be valid, it must meet the following conditions:

$$
\begin{aligned}
& X_{i} \in C, 1 \leq \mathrm{i} \leq n \\
& X_{i} \neq X_{j}, i \neq j, 1 \leq i, j \leq n \\
& X_{i}=X_{j}, i=j, 1 \leq i, j \leq n
\end{aligned}
$$

With this model, as well as binary encoding problem does not occur, cutting and mutation operators also can be used. The study focuses on the standard traveling salesman problem (symmetrical) and since there is a path between any two cities, we are not concerned about the validity of the links.

route between the city $\mathrm{i}$ to $\mathrm{j}$. So the cost of each chromosome can result in the following:

$$
\operatorname{Cost}_{1}(\mathrm{X})=\sum_{\mathrm{i}=1}^{\mathrm{n}-1} \mathrm{D}\left(\mathrm{X}_{\mathrm{i}}, \mathrm{X}_{\mathrm{i}+1}\right)+\mathrm{D}\left(\mathrm{X}_{\mathrm{n}}, \mathrm{X}_{1}\right)
$$

Thus, each chromosome that its cost is lower is better.

Since standard TSP problem is intended, we have:

$$
\mathrm{D}\left(\mathrm{X}_{\mathrm{i}}, \mathrm{X}_{\mathrm{j}}\right)=\mathrm{D}\left(\mathrm{X}_{\mathrm{j}}, \mathrm{X}_{\mathrm{i}}\right)
$$

What has been mentioned is the common assessment that all articles and books have been introduced for the traveling salesman problem. But this function has a weakness that is shown in an example and by providing a new method we eliminate the disadvantage.

If you have two chromosomes as 8 and with common methods they are evaluated, both are equal to the value of 200 with the same fitness.

Although the fitness of the two chromosomes are equal, but the two chromosomes in order to go to the cities, and then in cost of route between the cities are different. If the two chromosomes are more closely considered, we can see that the chromosome (b) has a route at a cost of less than chromosomes (a). According to the philosophy of the genetic algorithm, the chromosome (b) is better than (a) because it has shorter routes, even although two or three routes in this chromosome has a very high cost. The cause of this issue is that by the cutting operators we can extract low- 
cost routes from chromosome (b) and use them to amend other chromosomes that it will increase the effectiveness of GA.

Now we would like to define evaluation function in a way that this important thing is met and between two chromosomes (a) and (b) there be a difference. To be able to make the difference between two chromosomes, the first proposal is the product of cost of paths in addition to the sum of their costs when evaluating the chromosomes. The reason for use of the product of cost of routes is this that the product of large numbers is greater than smaller numbers. Accordingly, we prefer that the product of cost of chromosomes' path is less that this indicates that the chromosome is of low-cost routes or indeed a series of lowcost and effective routes exist in this chromosome. The proposed cost function in the first place will be as follows:

$$
\operatorname{Cost}_{2}(X)=\prod_{i=1}^{n-1} D\left(X_{i}, X_{i+1}\right) \times D\left(X_{n}, X_{1}\right)
$$

On the other hand, the use of the product is possible only for problems with a small number of cities because the calculations volume gets too high and because we always want to get the answer as soon as possible, this suggestion has not reached the desired result. Finally to solve the problem of calculation volume of the multiplication, logarithm function can be used and thus multiplying turned to the addition. Since the monotonic function is a logarithmic function, it can keep the problem situation. So for the first proposal evaluation function, matrix of cost of route between the cities once at the beginning of the algorithm is taken and then the cost of routes of chromosomes are added together. Therefore, the proposed evaluation function will be as follows:

$$
\begin{gathered}
\widehat{D}=\log \mathrm{D} \\
\operatorname{Cost}_{3}(\mathrm{X})=\sum_{\mathrm{i}=1}^{\mathrm{n}-1} \widehat{\mathrm{D}}\left(\mathrm{X}_{\mathrm{i}}, \mathrm{X}_{\mathrm{i}+1}\right)+\widehat{\mathrm{D}}\left(\mathrm{X}_{\mathrm{n}}, \mathrm{X}_{1}\right)
\end{gathered}
$$

Use only a proposed assessment function has no good result, it is therefore important that both functions (the common and proposed proposal) to be used for evaluation. For this purpose, we use the following weight equation:

$$
\text { Cost }=\alpha \times \operatorname{Cost}_{1}+(1-\alpha) \times \operatorname{Cost}_{3}
$$

Where $\alpha$ is a constant between 0 and 1 that 0.7 is considered in this study. In this case, we could have the benefits of both functions simultaneously. So the chromosome is better that has less cost.

\section{Elitism}

Elitism in GA means that some of the best chromosomes in each generation are directly transferred to other generation. This would result in this that we consistently and over many generations have the best chromosomes. We in this study consider $5 \%$ of the population as the elites and they are transferred directly without any changes to the next generation.

\section{E. Selection operator}

One of the most important operators in GA is selection operator. The right choice of this operator has a great impact on the final result of GA. This operator is used to select a parent to reproduce. By choosing two parents to combine, we choose the area of the search space is explored in the next generation. Selection Operators are very numerous and some of them are: roulette wheel, rank-rating tournament [32].

Selection operator is very impressive on population convergence. Thus, according to the application that we have we should be careful in choosing the right operator. In this study because we wanted to postpone convergence, the tournament operator with size of 10 was used. In this method, a subset of the population people are randomly selected with specified size; then the chromosome that among those is with the lowest cost will be introduced as a parent.

\section{F. cutting operator}

Using cutting operation, we combined with two chromosomes to get better chromosome. So we try to define cutting operator so that the children of parents combined, has low-cost links of parents, so the children have a lower cost to the parents [33]. Therefore various cutting operators have been introduced $[17,23]$. Each of these operators is of advantages and disadvantages. In this study, based on the needs, the cutting operator introduced by M. Obitko [24] has been used to produce better chromosomes.

In this way the cut-off point will be selected randomly and then permutations of the first parent are copied until the cut-off point of the first child and then the second parent is searched from beginning and any symbol that in the child has not been copied, will be copied in the child. The combination method of two chromosomes in this way you can see in Figure 2.

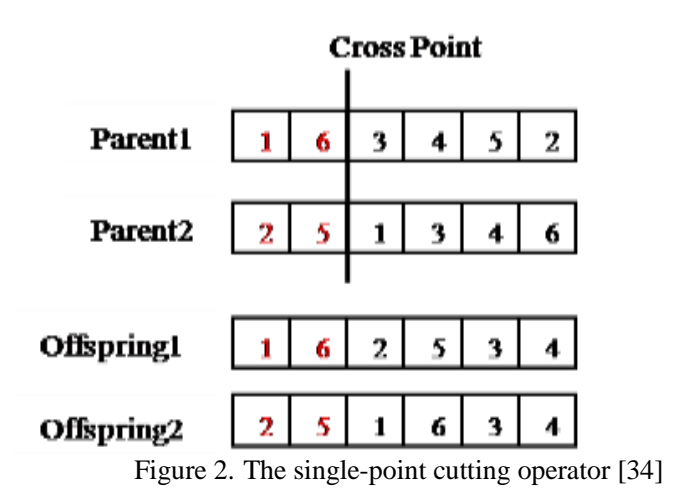

\section{G. mutation operator}

Mutation operator is the one that operates only on a chromosome and randomly change one of the genes in the chromosomes. One of the goals that we apply mutation operator, is increasing the diversity of population that makes up the population will not be soon convergent. 
In this study, the opposite window mutation is used. In this way two points on a random basis on are selected and then the gene sequence (numbers) between these two points gets reverse. Figure 3 shows the use of this operator on a chromosome.
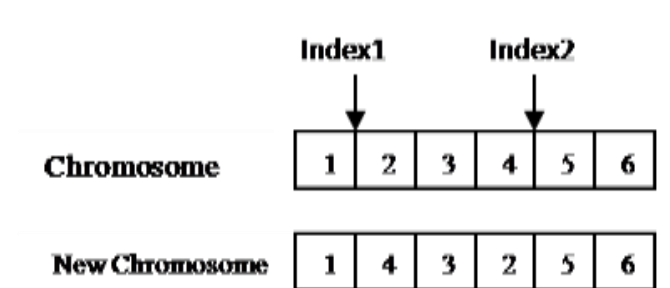

Figure 3.Reverse window mutation operator

Table 1.parameters of GA

\begin{tabular}{|c|c|c|c|c|}
\hline $\begin{array}{c}\text { Population } \\
\text { size }\end{array}$ & $\begin{array}{c}\text { Maximum } \\
\text { number of } \\
\text { evolution }\end{array}$ & $\begin{array}{c}\text { Cutting } \\
\text { probability }\end{array}$ & $\begin{array}{c}\text { Mutation } \\
\text { probability }\end{array}$ & \\
\hline 40 & 2000 & 0.7 & 0.2 & value \\
\hline
\end{tabular}

\section{H. Features of given GA}

We have used the genetic algorithms shown in Table 1 [35]. For this we have selected the mutation rate relatively high that we can control the convergence of the population.

To declare the convergence of the population we need to introduce a measure of stop. Stop criteria vary and they can be the number of produced generations, the standard deviation of the population, and optimized level for the ultimate solution or the past generations and in it the best chromosome has not changed. Since in this study the objective of demonstration is display of the effectiveness of the proposed evaluation function, we have considered the stop measure reaching the maximum number of evolution so that justice is observed. In this case, the best chromosome of the population would be the optimal solution.

\section{v. Simulation and presenting results}

All tests were conducted using a sample of current issues in TSPLIB. Results are from 10 times of the performance of each of the algorithms and the average on their answers. As seen in Table 2, the proposed algorithm compared to standard GA algorithm in the same conditions (in terms of structure, values and operating conditions) has found a better solution. Accordingly, it is possible to realize the extent to which the evaluation function definition can be effective in solving the problem and a small change in the evaluation function will result in better and more solutions.
Table 2-results of tests

\begin{tabular}{|c|c|c|c|c|}
\hline $\begin{array}{c}\text { The found } \\
\text { cycle } \\
\text { length by } \\
\text { proposes } \\
\text { method }\end{array}$ & $\begin{array}{c}\text { The found } \\
\text { cycle } \\
\text { length by } \\
\text { GA } \\
\text { standard }\end{array}$ & $\begin{array}{c}\text { Optimal } \\
\text { cycle } \\
\text { length }\end{array}$ & $\begin{array}{c}\text { Number of } \\
\text { cities }\end{array}$ & $\begin{array}{c}\text { Problem } \\
\text { name }\end{array}$ \\
\hline 8207.8 & 8230.1 & 7542 & 52 & Berlin52 \\
\hline 718.86 & 722.58 & 629 & 101 & Eill01 \\
\hline 462.11 & 462.11 & 426 & 51 & Eil51 \\
\hline 596.05 & 598.38 & 538 & 76 & Eil76 \\
\hline 25326 & 25680 & 22141 & 100 & KroB100 \\
\hline 115570 & 115880 & 108159 & 76 & Pr76 \\
\hline 1391.8 & 1399.8 & 1211 & 99 & Rat99 \\
\hline
\end{tabular}

\section{vi. Conclusion and recommendations}

Traveling salesman problem, the problem is well known and classical in operational research. This can represent many problems and scientific activities that to solve needs optimization methods involving many variables and constraints are taken into account. Therefore, solving problems such as genetic algorithms, neural networks and frequency heuristic methods to overcome this problem are suggested. In this regard, meta-heuristic algorithms because of its simple structure and abilities that have been shown have been used by many researchers.

In this study, the improved genetic algorithm has been used to solve TSP that is different in evaluation function with standard genetic algorithm. The new evaluation function is a combination of evaluation function and applying one other operator on the length of routes that the result is providing the paths with lower costs.

The proposed method was evaluated on seven sets of standard graph and the results of which indicate the maximum reduction of $20 \%$ of route length in some graphs and also the average of loss of about $7 \%$ over the route on the entire experiments.

Including proposals that can be presented in order to improve the proposed method, is using multi-objective optimization methods that using them can be reach a better solution than a single objective mode.

\section{References}

[1] R. Matai, S. Singh and M. Lal Mittal. Traveling Salesman Problem: an Overview of Applications, Formulations, and Solution Approaches, Traveling Salesman Problem, Theory and Applications, Prof. Donald Davendra (Ed.), ISBN: 978-953-307-426-9, InTech, November, 2010.

[2] K. De and A. Chaudhuri. A Study of Traveling Salesman Problem Using Fuzzy Self Organizing Map, Traveling Salesman Problem, Theory and Applications, Prof. Donald Davendra (Ed.), ISBN: 978-953-307-426-9, InTech, November, 2010.

[3] Held, M., Karp, R.M.: A dynamic programming approach to sequencing problems. Journal of the Society for Industrial and Applied Mathematics. vol. 10, pp. 196-210 (1962). 
[4] A. Misevičius. Using Iterated Tabu Search for the Travelling Salesmen Problem. Informacines Technologijos ir Valdymas, No 32/3, 2004, s. 2940.

[5] Md. R. Hassan, Md. K. Hasan, M.M.A. Hashem, An Improved ACS Algorithm for the Solutions of Larger TSP Problems,Procs. of the 3rd International Conference on Electrical, Electronics and Computer Engineering (ICEECE 2003), pp. 201-206, Dhaka, Bangladesh, December 22-24, (2003).

[6] K. H. Hingrajiya, R. K. Gupta, G. S. Chandel, An Ant Colony Optimization Algorithm for Solving Travelling Salesman Problem, International Journal of Scientific and Research Publications, Volume 2, Issue 8, August 2012.

[7] L. Tiankun et al. An Improvement of the Ant Colony Optimization Algorithm for Solving Travelling Salesman Problem (TSP). in Wireless Communications, Networking and Mobile Computing, 2009. WiCom '09. 5th International Conference on. 2009.

[8] L. Bifan, W. Lipo and S. Wu. Ant Colony Optimization for the Traveling Salesman Problem Based on Ants with Memory. in Natural Computation, 2008. ICNC '08. Fourth International Conference on. 2008.

[9] L. Lijie, J. Shangyou, and Z. Ying. Improved Ant Colony Optimization for the Traveling Salesman Problem. in Intelligent Computation Technology and Automation (ICICTA), 2008 International Conference on. 2008.

[10] M. Hong-biao, W. Jiang, and R. Zi-hui. An Adaptive Dynamic Ant System Based on Acceleration for TSP. in Computational Intelligence and Security, 2009. CIS '09. International Conference on. 2009.

[11] X. Yan, C. Zhang, W. Luo, W. Li, W. Chen and H. Liu, Solve Traveling Salesman Problem Using Particle Swarm Optimization Algorithm, IJCSI International Journal of Computer Science Issues, Vol. 9, Issue 6, No 2, November 2012, pp. 264:271.

[12] C. Zhang, J. Sun, Y. Wang, Q. Yang, An Improved Discrete Particle Swarm Optimization Algorithm for TSP. Proceedings of Web Intelligence/IAT Workshops'2007, 2007, s. 35-38.

[13] M. Antosiewicz, G. Koloch, B. Kamiński, Choice of best possible metaheuristic algorithm for the travelling salesman problem with limited computational time: quality, uncertainty and speed, Journal of Theoretical and Applied Computer Science, Vol. 7, No. 1, 2013, pp. 46-55.

[14] C. W. Tsai, S. P. Tseng, M. C. Chiang, C. S. Yang, and T. P. Hong, "A High-Performance Genetic Algorithm: Using Traveling Salesman Problem as a Case," The Scientific World Journal, vol. 2014, Article ID 178621, 14 pages, 2014. doi:10.1155/2014/178621.

[15] J. H. Holland, Adaptation in natural and artificial systems, The University of Michigan press, 1975.

[16] R.M. Brady, Optimization Strategies Gleaned From Biological Evolution, Nature, 317, pp. 804-806.

[17] S. S. Ray, et al., "New Operators of Genetic Algorithms for Traveling Salesman Problem," Proc. Proceedings of the 17th International Conference on Pattern Recognition (ICPR'04), IEEE.

[18] R. Takahashi, "Solving the traveling salesman problem through genetic algorithms with changing crossover operators," Proceedings. Fourth International Conference on Machine Learning and Applications, 2005, vol., no., pp.6 pp.,, 15-17 Dec. 2005.

[19] R. Takahashi, "A Methodology of Extended Changing Crossover Operators to Solve the Traveling Salesman Problem," Fourth International Conference on Natural Computation, 2008. ICNC '08, vol.1, no., pp.263,269, 18-20 Oct. 2008.

[20] R. Yang, "Solving large travelling salesman problems with small populations," Second International Conference On Genetic Algorithms in Engineering Systems: Innovations and Applications, 1997. GALESIA 97. (Conf. Publ. No. 446), vol., no., pp.157,162, 2-4 Sep 1997.
[21] C. Zhou, J. Zheng, W. Li, "An Improved Heuristic Crossover Operator for TSP," Fourth International Conference on Natural Computation, 2008. ICNC '08, vol.1, no., pp.541,545, 18-20 Oct. 2008.

[22] K. Rajan and AK. Anilkumar, GENETICALLY MOTIVATED SEARCH ALGORITHM FOR SOLVING TRAVELLING SALESMAN PROBLEM, Vol. XVI: No. 1 \& 2 SB Academic Review 2009: 19-31.

[23] K. RANI and V. KUMAR, SOLVING TRAVELLING SALESMAN PROBLEM USING GENETIC ALGORITHM BASED ON HEURISTIC CROSSOVER AND MUTATION OPERATOR, International Journal of Research in Engineering \& Technology (IMPACT: IJRET), Vol. 2, Issue 2, Feb 2014, 27-34.

[24] P. Larranaga, C. M. H. Kuijpers, R. H. Murga, I. Inza, S. Dizdarevic: "Genetic Algorithms for the Travelling Salesman Problem: A Review of Representations and Operators", Artificial Intelligence Review. 13, 1999, $129-170$

[25] N. kumar, Karambir, R. Kumar, A GENETIC ALGORITHM APPROACH TO STUDY TRAVELLING SALESMAN PROBLEM, Journal of Global Research in Computer Science, Volume 3, No. 3, March 2012. pp:33-38.

[26] Zambito. The Traveling Salesman Problem: A Comprehensive Survey, Project for CSE 4080, 2006

[27] T. A. El-Mihoub, A. A. Hopgood, L. Nolle, and A. Battersby, "Hybrid genetic algorithms: A review", Engineering Letters, vol. 3, no. 2, pp. 124137, 2006 .

[28] P. D. Thanh, H. T. T. Binh and B. T. Lam, A Survey on Hybridizing Genetic Algorithm with Dynamic Programming for Solving the Traveling Salesman Problem, Trends in Innovative Computing, 2013.

[29] A. Abu-Srhan and E. Al Daoud, A Hybrid Algorithm Using a Genetic Algorithm and Cuckoo Search Algorithm to Solve the Traveling Salesman Problem and its Application to Multiple Sequence Alignment, International Journal of Advanced Science and Technology, Vol.61, (2013), pp.29-38.

[30] Z. Zukhri and I. V. Paputungan, A HYBRID OPTIMIZATION ALGORITHM BASED ON GENETIC ALGORITHM AND ANT COLONY OPTIMIZATION, International Journal of Artificial Intelligence \& Applications (IJAIA), Vol. 4, No. 5, September 2013.

[31] D. Kaur, M. M. Murugappan, Performance enhancement in solving traveling salesman problem using hybrid genetic algorithm. Fuzzy Information Processing Society, NAFIPS. 1-6, 2008.

[32] A. Gog, D. Dumitrescu, B. Hirsbrunner, "New selection operators based on genetic relatedness for evolutionary algorithms," IEEE Congress on Evolutionary Computation, 2007. CEC 2007, vol., no., pp.4610,4614, 25-28 Sept. 2007.

[33] Y. Nagata, "Criteria for designing crossovers for TSP," Congress on Evolutionary Computation, 2004. CEC2004, vol.2, no., pp.1465,1472 Vol.2, 19-23 June 2004

[34] M. Obitko, "Introduction to Genetic Algorithms," available in http://www.obitko.com/tutorials/genetic-algorithms/crossovermutation.php.

[35] G. Reinelt, "TSPLIB - A Traveling Salesman Problem Library," ORSA Journal on Computing, no 3, 1991, pp. 376 - 384. 